\title{
Identifying student resources for understanding kinematics
}

\author{
Cheyenne Broadfoot \\ Department of Physics, University of Washington, 18115 Campus Way NE, Bothell, WA, 98011 \\ Brynna Hansen, Amy D. Robertson \\ Department of Physics, Seattle Pacific University, 3307 Third Ave W, Seattle, WA, 98119 \\ Lisa Goodhew \\ Department of Physics, University of Washington, 1410 NE Campus Parkway, Seattle, WA, 98195
}

\begin{abstract}
Physics education research (PER) studies of students' understanding of kinematics have largely been focused on difficulties and misconceptions - ideas that act as barriers to student understanding of formal physics. Our research takes an alternative perspective on students' ideas. We offer insight into student resources for understanding kinematics - ideas that we consider to be the "beginnings" of a more sophisticated scientific understanding of motion. Our analysis of 582 written student responses to several kinematics questions highlights four common resources students use when justifying their solutions to different problems. In this paper, we elaborate on these four resources and give examples from our preliminary research.
\end{abstract}




\section{INTRODUCTION}

An important contribution of physics education research (PER) has been providing instructors with knowledge of student ideas (KSI) [1], which supports instructors in anticipating their students' thinking and planning instruction accordingly. To date, much of this research is focused on identifying incorrect patterns in student reasoning. This general trend is also true of research focusing on student understanding of basic kinematics principles. For example, some studies have found that students have difficulty understanding qualitative and quantitative definitions of acceleration and velocity [2,3], as well as how the two quantities are related. Others report that students fail to differentiate between position, velocity, and acceleration $[4,5]$. Still others make connections between difficulties in interpretation and problem-solving and limitations in specific mathematical skills required for these tasks $[5,6]$. These studies reflect a stance towards errors as reflecting incorrect ideas. In this paper, we bring the theoretical lens of resources [7-10] to the effort of characterizing common, potentially fruitful ideas that students use to answer kinematics questions. Our aim is to contribute to instructors' KSI by supplementing existing misconceptions-based research on students' common ideas about kinematics.

Using our analysis of 582 written university student responses to three different kinematics problems, we answer the question: What are some of the common conceptual resources that students use to reason about kinematics? We identified four recurring resources: the "definition," "magnitude," "direction," and "gravity" resources. In defining and illustrating these four resources, we hope to provide instructors with concrete information about students' ideas and to offer an example of how instructors can identify student resources in their own classrooms, thus supporting a resources-oriented approach to teaching kinematics.

\section{THEORETICAL FRAMEWORK}

Resources theory conceptualizes thinking as in-themoment activation of resources to construct explanations, arguments, and concepts [7-9]. Resources are derived from students' prior experience and learning, and they are sensible - i.e., students have good reason for using them as they do $[8,9]$. This theoretical framework suggests that learning involves refining, limiting or extending the context for activation of, or strengthening networks of connection between resources $[7,10]$. That is, the process of learning involves building from existing ideas and understandings.

In this paper, we primarily draw on resource theory's orientation to student thinking, analyzing students' responses to written questions about kinematics for ideas that are resourceful and fruitful for scientific thinking. We are guided by the assumption that learning and instruction can build from the resources students bring, and a number of our choices are guided by our aim to support instructional planning toward this end. For example, we report resources that are activated in response to multiple questions, for many students, expecting that these may inform instructional predictions. We attend specifically to student resources for understanding kinematics, and the resources we name in Section IV are at the grain-size of ideas - what students say or write down. This choice is consistent with resources that have been reported in the literature - e.g., a small "car reacts twice as much" as a big truck in a collision [7] - but is larger than the grain size of p-prims and other kinds of resources.

\section{RESEARCH METHODS AND CONTEXT}

The resources we report in this paper were identified in written student responses to three kinematics tasks - the ball on ramp explain question, the circular track explain question, and the add specificity kinematics question, all shown in Figure 1. These questions were designed to elicit student reasoning about velocity and acceleration. The first two were designed to explore student thinking about the relationship between velocity and acceleration. One way to answer these two questions is by applying the definition of acceleration as the change in velocity per unit time for pairs of instants shown. For example, in the ball on ramp explain question, the ball slows down as it moves in a straight line up the ramp; thus, the change in velocity and acceleration vectors point directly opposite the velocity vector at each point. The change in velocity is due to a constant gravitational force, and so the magnitude of the acceleration is also constant. Similar reasoning could be constructed for the circular track question. The third question was designed to capture what we see as one of the central goals of kinematics instruction: to add precision to the ways in which we describe motion. One way a student might add specificity to the first statement in the question would be to say, "A bowling ball falls out of a moving airplane. The ball maintains its speed in the horizontal direction and speeds up vertically because the net force on the ball is straight down. Thus, it follows a curved trajectory like the one in the figure at right."

We analyzed a total of 582 written responses from introductory physics courses at three US colleges and universities: Bellevue College, George Mason University (GMU), and the University of Washington (UW). (Sample sizes by question and college/university are given in Table I.) Students answered the questions on homework, exams, or quizzes. The course response rates were $84.3 \%$ for questions asked at Bellevue College, $50 \%$ at GMU, and between $89.1 \%$ and $100 \%$ at UW.

The racial and/or ethnic demographics for the colleges/universities in our study compared to all college/ university students are shown in Figure 2. Figure 2 suggests that our study is oversampling from colleges/universities that serve Asian (including Asian American) students and under- 


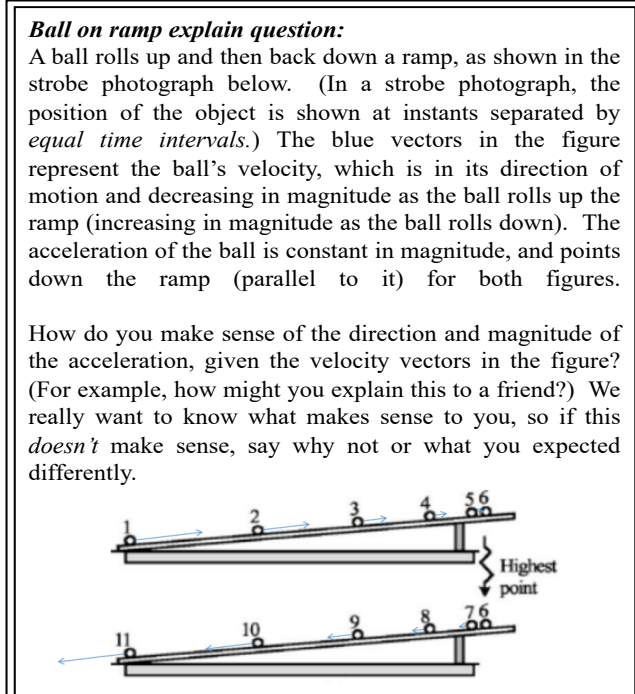

\section{Circular track explain question:}

A car moves at constant speed along a closed, oval track, as shown in the top-view diagram (looking down at the track from above) below. The blue vectors in the diagram represent the car's acceleration, which is perpendicular to the car's velocity vector at points $\mathrm{A}$ through $\mathrm{F}$ and changes in magnitude in the ways reflected in the diagram. The velocity of the car is the same magnitude for all five points and always points tangent to the curve.

How do you make sense of the direction and magnitude of the velocity and acceleration in this scenario? (For example, how might you explain this to a friend?) We really want to know what makes sense to you, so if this doesn't make sense, say why not or what you expected differently.

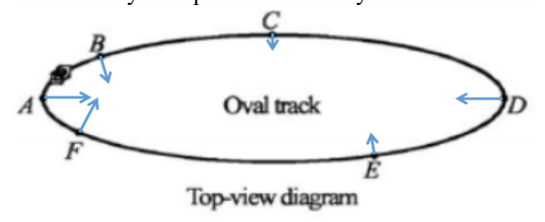

Add specificity kinematics question:

The concepts of speed/velocity, acceleration, and force are often used to describe or explain motion and changes in motion. Sometimes, these concepts add precision; for example, if I want to add precision to the description "a ball falls from the table," I might say that the ball's speed changes as it falls, and I can quantify this change in speed using acceleration due to gravity. Or I can say that the gravitational force is what changes the ball's speed by pushing it. For each of the following situations, add precision to the description or offer an explanation for the object's motion, using the concepts of speed/velocity, acceleration, and/or force:

a. A bowling ball falls

out of a moving an

airplane

follows

trajectory shown in

the figure at right.

b. A sports car passes a bus on the highway. c. A box slides to a stop on the floor.

FIG. 1. Three kinematics questions used in this study. Figures in the ball-on-ramp explain and circular track explain questions reproduced with permission from P. S. Shaffer and L. C. McDermott, "A research-based approach to improving student understanding of the vector nature of kinematical concepts," American Journal of Physics 73(10), 921, with the permission of the American Association of Physics Teachers. Figure in the add specificity kinematics question reproduced with permission from D. Hestenes, M. Wells, and G. Swackhamer, "Force Concept Inventory," The Physics Teacher 30, 141, with the permission of the American Association of Physics Teachers.

sampling from colleges/universities that serve Hispanic/Latinx and Black students. In addition, the median parental income of the students at colleges/universities in our study is higher than the national average. This over- and under-sampling limits the generalizability of our results, and it contributes to a broader trend identified by Kanim and Cid [11], who highlighted that PER in the US has historically oversampled from a small slice of the population of collegebound students (in particular, white, wealthy, mathematically-prepared students). This oversampling has directed the benefits of research and curriculum development to students in these groups and has constructed such students as an implicit norm, reinforcing deficit models of students who are not white, wealthy, and mathematically prepared. One limitation of Figure 2 and our comparison of parental income is that they are based on university-level data, rather than data at the level of our sample. We are accumulating sample-level data, but until we know more about what constitutes a representative sample of introductory physics students, sample-level data does not allow for comparisons to a national average.

We used responses to the three questions in Figure 1 to create an emergent coding scheme [12] that captured some of the common resources students used to reason about kinematics. To construct this scheme, authors $\mathrm{CB}$ and $\mathrm{BH}$, in consultation with ADR, conducted preliminary analyses of student responses to each question, looking for ideas that we considered to be continuous with relevant (kinematical) formal physics concepts, even if not stated in formal terms. We used these preliminary analyses to identify patterns in student reasoning across questions, foregrounding a model of generalizability that emphasizes recurrence across multiple sources of heterogeneity [13]. This process produced a final scheme with four resources.

Authors $\mathrm{CB}$ and $\mathrm{BH}$ independently coded student responses to the three questions. Resources are often activated in concert; thus, a single response could receive no code, one code, or many codes. As a measure of inter-rater agreement, we took the normalized difference between the total number of possible codes and the total number of disagreements between the two coders. We used percentage agreement rather than a standard statistical measure of

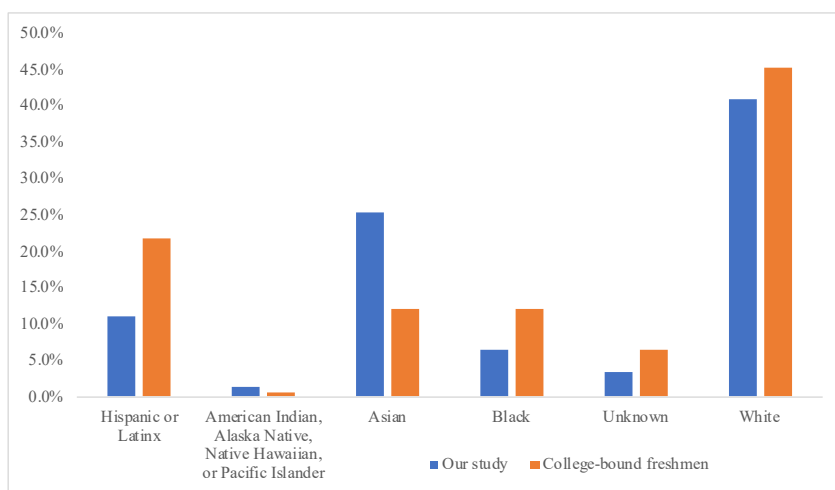

FIG. 2. Racial and/or ethnic demographics of institutions in our sample (blue) versus all college-bound freshmen (orange). Blue bars were constructed using demographic data provided by offices of institutional research or institutional websites, weighted by sample size. Orange bars were constructed using data from Kanim and Cid [11]. As explained by activist Kat Lazo [16], neither Hispanic nor Latinx are racial groups, and these two identities are not the same. "Hispanic" is a descriptor for people of Spanish-speaking origins, and "Latinx" is a descriptor for people with origins in Latin America. The former focuses on language, the latter on geographic location. 
agreement (e.g., Cohen's kappa) because our codes are not independent or mutually exclusive $[14,15]$. The original percentage agreement for the full data set was $82.3 \%$. All disagreements were resolved through discussion, and thus the percentages reported in Table I reflect complete agreement between two coders.

\section{RESULTS}

Here, we discuss four resources for understanding kinematics that were common in our data set. To be considered common, a resource had to appear in at least $10 \%$ of student responses and in responses to multiple questions.

The names of the resources emphasize their continuity with formal physics; that is, they are phrased in terms that highlight what we saw as fruitful about patterns in student reasoning. These resources were marshaled in the service of both correct and incorrect answers; students' responses did not need to be correct overall to be assigned a code.

Table 1 shows the prevalence of each resource across questions and samples. We discuss the variability in student use of resources in our Discussion.

TABLE I. Percentages of responses in each sample assigned each resource code.

\begin{tabular}{lcccc}
\hline \hline Sample & A & B & C & D \\
\hline Ball on ramp explain $\mathrm{Q}$ & & & & \\
Bellevue College $(N=59)$ & $3 \%$ & $2 \%$ & $8 \%$ & $51 \%$ \\
GMU $(N=36)$ & $22 \%$ & $11 \%$ & $19 \%$ & $25 \%$ \\
UW $(N=175)$ & $38 \%$ & $34 \%$ & $50 \%$ & $5 \%$ \\
Circular track explain Q & & & & \\
Bellevue College $(N=59)$ & $48 \%$ & $63 \%$ & $52 \%$ & $0 \%$ \\
UW $(N=155)$ & $27 \%$ & $25 \%$ & $10 \%$ & $0 \%$ \\
Add specificity kinematics $\mathrm{Q}$ & & & & \\
Bellevue College $(N=59)$ & $37 \%$ & $3 \%$ & $0 \%$ & $61 \%$ \\
GMU $(N=39)$ & $31 \%$ & $8 \%$ & $5 \%$ & $51 \%$ \\
\hline \hline
\end{tabular}

\section{A. Acceleration is present when there is a change in the velocity of an object (the "definition" resource).}

Many student responses indicated that an object is experiencing acceleration when the velocity of the object is changing, and we assigned these the "definition" resource code. As shown in Table I, this resource appeared in roughly $20-40 \%$ of the responses from the universities that were sampled, across questions, suggesting that the "definition" resource may be a common way for students to make sense of the questions about acceleration.

Responses using this resource show that students' reasoning is often continuous with the formal definition of acceleration as a change in velocity (magnitude and/or direction) of an object. For example, in response to the circular track question, one student wrote, "Because the object does not stay in motion in a straight line, an acceleration must be changing the direction of velocity." We also assigned the "definition" resource code to responses that stated that acceleration was not present when there was no change in an object's speed or direction. For example, in the add specificity question, one student states: "A sports car passes a bus on a highway. There is no mention of change in velocity, therefore we can assume no acceleration occurs."

\section{B. The magnitude of acceleration is related to the magnitude of the change in velocity (the "magnitude" resource)}

We assigned this code to student responses that identified a relationship between the magnitude of an object's change in velocity and the magnitude of its acceleration. In contrast to the "definition" resource, which states that acceleration exists when there is a change in velocity, the "magnitude" resource relates the magnitude of acceleration to the magnitude of the change in velocity. This resource was common in responses to the ball on ramp question (11-35\%) and the circular track question (25-63\%). The add specificity question rarely elicited responses that used this resource.

As one example, a student wrote the following in response to the circular track question: "At points with a higher rate of change in direction... magnitude of centripetal acceleration (written as $\mathbf{a}_{\mathrm{c}}$ ) is larger." This example of the "magnitude" resource explicitly states that the magnitude of centripetal acceleration is larger because there is a greater change in direction (of velocity) when the curve of the track is tighter. Students also used this resource to connect constant change in velocity to constant acceleration. For example, one response from the ball on ramp question reads, "The velocity vector always changes by a fixed amount no matter [whether the speed is] increasing or decreasing. Therefore, the magnitude of acceleration is held constant."

\section{The directional relationship between an object's velocity and acceleration determines what is observed about its speed (the "direction" resource).}

The "direction" resource connects a visual, intuitive indicator (i.e., speeding up, slowing down or constant speed) to the directional relationship of the velocity and acceleration vectors. Responses that explicitly state how the directional relationship between velocity and acceleration vectors changes the speed were given this code. For example, one student said in their answer to the ball on ramp question that "... the velocity is decreasing therefore the acceleration vector's magnitude will be pointing in the opposite direction..." and "when the ball moves down the ramp, the acceleration vector is pointed in the same direction because the ball is speeding up." Other examples of this resource associated perpendicular velocity and acceleration vectors with constant speed, such as this response to the circular track question: "the acceleration vector is pointing perpendicular to the velocity vector at each point so that the car remains at a constant speed, but changes direction." Table I shows that this resource was particularly prevalent in the ball on ramp question (19-50\%) and circular track question (10-52\%). Similar to the "magnitude resource," this 
resource was rarely used in responses to the add specificity question.

\section{Gravity changes the speed of objects (the "gravity" resource).}

The "gravity" resource recognizes gravity as an agent that changes an object's velocity or recognizes gravity as a source of acceleration. Table I shows that students frequently used the "gravity" resource in responses to the ball on ramp question (25-51\%) and add specificity question (51-61\%), but not the circular track question. Student answers that identified gravity as an initiator for object's change in speed were given this code, as in this response to the add specificity question: "The bowling ball's velocity increases after leaving the plane in a constant acceleration due to gravity." Another student responded to the ball on ramp question by stating: "Gravity slows the ball down when it is moving against it i.e. up the ramp and speeds the ball up when it moves with it i.e. down the ramp." Both responses point to gravity as a cause of changing speed; the first response also acknowledges that gravity causes an object's speed to change at a constant rate. As these responses show, the "gravity" resource appropriately connects a familiar agent gravity - to the abstract concept of acceleration.

\section{DISCUSSION}

In this paper, we answer the question "What are some of the common conceptual resources that students use to answer kinematics questions?" by identifying four resources that were common among student responses to conceptual kinematics questions in our sample. The first three resources break down the concept of acceleration into pieces that are salient to students. In particular, student responses draw on the definition of acceleration as a change in velocity, the relationship between the magnitudes of acceleration and the change in an object's velocity, and the dependency of observable change in an object's speed on the relative directions of velocity and acceleration. Whereas the literature suggests that many students "fai[1] to discriminate between the concepts of velocity and acceleration" [3] (see also [17]), the results of our study - particularly resources A through $\mathrm{C}$ - suggest that students are marshaling pieces of the relationship between velocity and acceleration to explain and predict the motion of objects. Further, our analysis may offer a re-framing of some of the difficulties reported in the literature, such as the pattern that students "mistakenly assum $[\mathrm{e}]$ that the acceleration is zero because the speed is constant" [17]. This assumption is consistent with student use of resource A, and so our work may help position this idea as a productive beginning.

In responses that draw on the "gravity" resource, students go further than simply naming gravity as a force; they also focus on gravity as an active agent that changes the speed of objects. The "gravity" resource may be a way for students to relate their lived experience of gravity to formal physics notions of velocity and acceleration. In fact, some examples of the "gravity" resource are concrete instantiations of the "definition," "magnitude," and "direction" resources - e.g., responses that say the change in velocity of the ball is constant because gravity is constant.

Our study frames student ideas as the beginnings of sophisticated scientific thinking rather than as deficits or obstacles to learning. Though some of the responses instantiating resources A-D were canonically incorrect, they demonstrated pieces of canonical concepts that have the potential to be built on as students continue to learn and develop deeper understandings of kinematics concepts. It is important to acknowledge that while our resources are derived from student responses from various colleges, they may not be representative of the population of physics students, as seen in the demographic data presented in Section III. The ways in which population variability will change results like these is an open question [11].

Resources theory predicts that resource activation is context-dependent, such that certain resources may be more likely to be elicited in specific contexts, including the questions asked and the settings in which they are asked [710]. This prediction bears out in the frequencies of the four resources we identified (Table I). Notably, students at Bellevue College used the "gravity" resource at higher frequency than students at GMU or UW for the ball on ramp explain question, and the other three resources at lower frequency. Students at Bellevue College also used the "definition," "magnitude," and "direction" resources at higher frequency than students at GMU in response to the circular track question. The source of these differences is not known and could be attributed to a number of factors. As one example, instruction at Bellevue College could have focused largely on gravity, explaining the high percentage of students who identified resource D (the gravity resource).

Despite the variability, there are some trends that are instructionally relevant. For example, the "definition" resource was common across all three questions, showing that a variety of questions can elicit ideas about acceleration as a change in velocity. The "magnitude and "direction" resources were both more common among the ball on ramp question and the circular track question. This suggests to us that these questions may provide instructors with targeted opportunities to elicit student ideas about how the magnitude or direction of an object's acceleration relates to its speed or velocity at different times.

\section{ACKNOWLEDGMENTS}

This work was supported in part by the National Science Foundation under grant numbers $1914603 \& 1914572$. The authors wish to thank Paula R. L. Heron, Rachel E. Scherr, Raphael Mondesir, Lauren Bauman, Jonathan Corcoran, and McKensie Mack for offering helpful feedback on earlier versions of this paper, and all those who helped with data collection. 
[1] L. Shulman, Harv. Educ. Rev. 57, 1 (1987).

[2] A. Jones, Res. Sci. Ed. 13, 95 (1983).

[3] D. E. Trowbridge and L. C. McDermott, Am. J. Phys. 49, 242 (1981).

[4] R. J. Beichner, Am. J. Phys. 62, 750 (1994).

[5] H. Taylor and M. Loverude, in Proceedings of the 2018 Physics Education Research Conference, edited by A. L. Traxler, Y. Cao, and S. F. Wolf

[6] M. F. Tasar, ZDM Math. Ed. 42, 469 (2010).

[7] D. Hammer, Am. J. Phys. 69, S52 (2000).

[8] J.P. Smith, A.A. diSessa, and J. Roschelle, J. Learn. Sci. 3, 2 (1993).

[9] A. A. diSessa, Cognition Instruct. 10, 2 \& 3 (1993).

[10] D. Hammer, A. Elby, R.E. Scherr, and E.F. Redish, in Transfer of Learning from a Modern Multidisciplinary Perspective, edited by J.P. Mestre (Information Age Publishing, Greenwich, 2005), p. 89-119.

[11] https://arxiv.org/abs/1710.02598. Retrieved 4/29/2020.

[12] K. Krippendorff, Content Analysis: An Introduction to Its Methodology (Sage, Thousand Oaks, CA, 2013), $3^{\text {rd }}$ ed.

[13] T. D. Cook, Educ. Eval. Policy Anal. 24, 175 (2002).

[14] M. L. McHugh, Biochem. Medica 22, 276 (2012).

[15] J. Cohen, Educ. Psychol. Meas. 20, 37 (1960).

[16] https://www.vox.com/2016/8/28/12658908/latino-hispanicrace-ethnicity-explained. Retrieved 5/19/2020.

[17] P. S. Shaffer and L. C. McDermott, Am. J. Phys. 73, 921 (2005). 